\title{
High-Dose Amoxicillin with Clavulanate for the Treatment of Acute Otitis Media in Children
}

\author{
Chia-Huei Chu, ${ }^{1,2}$ Mao-Che Wang, ${ }^{1,2}$ Liang-Yu Lin,, ${ }^{3,4}$ Tzong-Yang Tu, ${ }^{1,2}$ \\ Chii-Yuan Huang, ${ }^{1,2}$ Wen-Huei Liao, ${ }^{1,2}$ Ching-Yin Ho, ${ }^{1,2}$ and An-Suey Shiao ${ }^{1,2}$ \\ ${ }^{1}$ Department of Otorhinolaryngology-Head and Neck Surgery, Taipei Veterans General Hospital, No. 201, Section 2, \\ Shih-Pai Road, Taipei 11217, Taiwan \\ ${ }^{2}$ Department of Otorhinolaryngology, National Yang-Ming University School of Medicine, No. 155, Section 2, Li-Nong Street, \\ Taipei 11217, Taiwan \\ ${ }^{3}$ Division of Endocrinology and Metabolism, Department of Medicine, Taipei Veterans General Hospital, No. 201, Section 2, \\ Shih-Pai Road, Taipei 11217, Taiwan \\ ${ }^{4}$ Department of Medicine, National Yang-Ming University School of Medicine, No. 155, Section 2, Li-Nong Street, Taipei 11172, Taiwan \\ ${ }^{5}$ Institute of Pharmacology, National Yang-Ming University School of Medicine, No. 155, Section 2, Li-Nong Street, Taipei 11217, Taiwan \\ Correspondence should be addressed to An-Suey Shiao; asshiao@gmail.com
}

Received 23 November 2013; Accepted 22 December 2013; Published 6 January 2014

Academic Editors: J. M. Coticchia, R. Matalon, C. O’Morain, and P. A. Schachern

Copyright (C) 2014 Chia-Huei Chu et al. This is an open access article distributed under the Creative Commons Attribution License, which permits unrestricted use, distribution, and reproduction in any medium, provided the original work is properly cited.

\begin{abstract}
Objective. This study uses the acute otitis media clinical practice guideline proposed in 2004 as a reference to evaluate whether antibiotics doses that are in line with the recommendations lead to better prognosis. The study also attempts to clarify possible factors that influence the outcome. Study Design. Retrospective cohort study. Subjects and Methods. A total of 400 children with acute otitis media were enrolled. The dosage of amoxicillin was considered to be appropriate when in accord with clinical practice guidelines, that is, $80-90 \mathrm{mg} / \mathrm{kg} /$ day. The outcome was defined according to the description of tympanic membrane on medical records. Multivariate logistic regression was used to analyze the relationship between antibiotic dosage and prognosis after adjusting for baseline factors. Results. The majority of prescriptions were under dosage (89.1\%) but it was not noticeably associated with outcome $(P=0.41)$. The correlation between under dosage and poor prognosis was significant in children below $20 \mathrm{~kg}$ with bilateral acute otitis media (odds ratio 1.63; 95\% CI 1.02-2.59, $P=0.04$ ). Conclusion. Treating acute otitis media in children, high-dose amoxicillin with clavulanate as recommended in the clinical practice guideline was superior to conventional doses only in children under $20 \mathrm{~kg}$ with bilateral diseases.
\end{abstract}

\section{Introduction}

Acute otitis media (AOM) is one of the common childhood infections, occurring often after acute upper respiratory tract infections. It is also the leading cause of clinic visits by children and the most frequent reason that doctors prescribe antibiotics [1-3]. According to insurance statistics, AOMrelated outpatient services in the US were as high as 16 million in 2000 , and $80 \%$ of the cases were prescribed with antibiotics. It had been reported that the estimated annual medical expenditure related to AOM is about $\$ 3.8$ billion to $\$ 5.3$ billion in the US $[4,5]$. The total cost per AOM episode ranged from $€ 332.00$ to $€ 752.49$ in several European countries [6]. In addition to medical expense from physicians' consultation and medications, AOM indirectly causes the loss of children's school time and caregivers' work time and income [7].

To reduce medical costs while maintaining high standards of medical quality, the American Academy of Pediatrics, the American Academy of Otolaryngology-Head and Neck Surgery, and the American Academy of Family Physicians endorsed an evidence-based comprehensive review, the AOM Clinical Practice Guidelines: Diagnosis and Management of AOM, which was published in May 2004 [8]. The guidelines emphasize the importance of correct diagnosis and 
provide suggestions for initial treatment of children between the age of 2 months and 12 years with uncomplicated AOM.

Amoxicillin with or without $\beta$-lactamase inhibitor is the mainstream choice in the treatment of AOM, but information on dosage comparisons of antibiotics and research on true clinical outcome is limited [9-11]. In the past, most studies on AOM focused on epidemiological surveys, laboratory bacteriological reports, and the therapeutic effects of different antibiotics $[12,13]$. Experts have published different viewpoints on the suggestions in the clinical practice guidelines, such as when to provide antibiotic treatment, how to give an adequate dose, and the proper duration of treatment. As most of the evidence comes from bacteriological research in laboratories and a small number of samples in clinical studies, higher level evidence is lacking [14-17]. Therefore, doctors still do not have a consensus on these issues [18].

This study aimed to evaluate whether physicians' prescriptions are in accordance with the dosage guidelines and whether high-dose amoxicillin with clavulanate is associated with better outcomes in pediatric AOM.

\section{Subjects and Methods}

2.1. Subjects. We conducted a retrospective cohort study from January 1, 2005, to December 31, 2008. Children aged 2 months to 12 years who were diagnosed with acute otitis media according to the diagnosis code (382.00) in the International Classification of Diseases, Ninth Revision, Clinical Modification (ICD-9-CM), were enrolled. Those who had been coded with any anatomic or genetic abnormalities, such as craniofacial anomalies or Down syndrome, or immune deficiencies were excluded. Children with histories of recurrent AOM (three or more previous episodes of AOM within 12 months), those who had undergone any middle ear or inner ear procedure, those who had only one visit, or those missing records were excluded. Their age, gender, weight, diagnosis date, unilateral or bilateral disease, and details in antibiotic prescriptions were reviewed.

2.2. Methods. Antibiotics prescriptions that contained amoxicillin with clavulanate (amoxicillin $400 \mathrm{mg}$ with clavulanate $57 \mathrm{mg} / 5 \mathrm{~mL}$ ) were reviewed in detail. Dosage was considered to be correct, that is, high dose, if the amoxicillin component was within $\pm 10 \%$ of the guideline suggestions. If the dosage was outside the $\pm 10 \%$ range, it was considered either overor under dose. The recommended dose of the amoxicillin component is $80-90 \mathrm{mg} / \mathrm{kg} /$ day. However, for children over $20 \mathrm{~kg}$, this might correspond to a dosage that surpasses the common clinical dosage for adults of $1500 \mathrm{mg} /$ day. Therefore, a daily dosage of $1500 \mathrm{mg}$ amoxicillin for children over $20 \mathrm{~kg}$ was considered to be acceptable.

To evaluate the relationship between amoxicillin dose and AOM prognosis, study analysis focused on the comparison of two groups: those who were prescribed with an amoxicillin dose that was in agreement with the guideline recommendations $(80-90 \mathrm{mg} / \mathrm{kg} /$ day) those who were not.

The assessment of treatment outcome was based on medical records within 14 days after the antibiotic prescription expiry date. In our institute, the middle ear status in children was mainly evaluated by telescope examination by a licensed otology specialist which had been demonstrated to have the highest sensitivity (97.8\%) and specificity (100.0\%) [19]. Successful control was defined as a medical record of an eardrum that either was normal or showed otitis media with effusion (OME). Failed control was defined as improvement in only one of two affected ears or a change in antibiotics before the end of the treatment period (with the reason for changing antibiotics being failure to control illness rather than side effects).

\section{Statistical Analysis}

Continuous variables such as age and body weight are presented as mean and standard deviation. Gender, single-ear or bilateral disease, and illness season were categorical variables and represented as numbers and percentages. To analyze the possible prognostic factors, we used a $t$-test and chisquare test for univariate analysis. All covariates in univariate analyses along with the two basic demographic variables (gender and weight) were then included in a binary logistic regression model. All statistical analyses were carried out with IBM SPSS statistical software version 14.0 for Windows (IBM Corp., New York, USA). Statistical significance was defined as a $P$ value of less than 0.05 .

\section{Results}

4.1. Clinical Characteristics of the Study Population. A total of 400 medical records with diagnosis code 382.00 were reviewed. There were 94 children with complicated AOM (i.e., clinical recurrence of AOM within 30 days, histories of recurrent AOM, AOM with underlying chronic otitis media with effusion, any underling anomalies that may alter the course of AOM such as cleft palate, genetic problems such as Down syndrome, immunodeficiencies, and status after any middle or inner ear surgery); 59 had only one visit, 40 had incomplete record, 15 were prescribed with cephalosporin or sulfa drugs, and 27 were prescribed with amoxicillin. One hundred and sixty five children treated with amoxicillin with clavulanate were included into analysis. The average age of the 165 enrolled patients was 4.91 years old ( $0.28-11.72$ years old), the average weight was $19.36 \mathrm{~kg}(7.50-48.0 \mathrm{~kg})$, and 94 were boys (57\%). Most illnesses occurred in spring (31.5\%) and autumn (28.5\%). Single-ear AOM occurred in 81 participants (49.1\%) (Table 1).

4.2. Antibiotic Dosage. Eighteen in 165 (10.9\%) prescriptions were in accord with the recommendations of the clinical practice guidelines. Underdosage was extremely common (89.1\%). None of the prescriptions exceeded the upper limit of $90 \mathrm{mg} / \mathrm{kg} /$ day. Overall, the average dose of the amoxicillin component was $45.5 \mathrm{mg} / \mathrm{kg} /$ day, which was far lower than the guideline suggestion. However, 86 (52.1\%) prescriptions were within the range of $40-50 \mathrm{mg} / \mathrm{kg} /$ day, the traditional usage for amoxicillin. In other words, half of the physicians indeed followed the conventional dosage. 
TABle 1: Demographic data of 165 children.

\begin{tabular}{lc}
\hline Characteristic & Number $(\%)$ \\
\hline Age: mean $4.91 \pm 2.52(\mathrm{y} / \mathrm{o})$ & \\
Body weight: mean $19.36 \pm 7.52(\mathrm{~kg})$ & \\
Gender & $94(57.0)$ \\
$\quad$ Boy & $71(43.0)$ \\
$\quad$ Girl & \\
Illness season & $52(31.5)$ \\
$\quad$ Spring & $28(17.0)$ \\
Summer & $47(28.5)$ \\
$\quad$ Autumn & $38(23.0)$ \\
$\quad$ Winter & \\
Single or bilateral disease & $81(49.1)$ \\
Single-sided AOM & $84(50 / 9)$ \\
\hline Bilateral AOM &
\end{tabular}

Abbreviations: AOM: acute otitis media.

TABLE 2: Treatment outcome of acute otitis media.

\begin{tabular}{lcccccc}
\hline & \multicolumn{2}{c}{ Overall } & \multicolumn{2}{c}{ High dose } & \multicolumn{2}{c}{ Underdose } \\
& $n$ & $(\%)$ & $n$ & $(\%)$ & $n$ & $(\%)$ \\
\hline Successful control & 121 & $(73.3)$ & 15 & $(83.3)$ & 106 & $(72.1)$ \\
$\quad$ Eardrum normal & 75 & $(45.5)$ & 10 & $(55.6)$ & 51 & $(34.7)$ \\
$\quad$ OME & 70 & $(42.4)$ & 5 & $(27.8)$ & 55 & $(37.4)$ \\
Failed control & 44 & $(26.7)$ & 3 & $(16.7)^{*}$ & 41 & $(27.9)^{*}$ \\
\hline Sum & 165 & & 18 & & 147 & \\
\hline
\end{tabular}

Abbreviations: OME: otitis media with effusion. ${ }^{*}$ Fisher exact test, $P=0.41$.

4.3. Antibiotic Dosage and Treatment Outcome. Successful control was achieved in 121 patients $(73.3 \%)$ while the remaining $26.7 \%$ had failed control. Fewer patients who were given the correct dose (i.e., high-dose amoxicillin) had poor AOM prognosis (16.7\% versus $27.9 \%)$, but this result was not significant (Fisher exact test, $P=0.41$ ) (Table 2).

4.4. Factors Affecting Treatment Outcome. In univariate analysis, only disease in autumn/winter was associated with a poor prognosis $(P=0.03)$. Age $(P=0.22)$, gender $(P=$ $0.07)$, and body weight $(P=0.25)$ were not associated with treatment outcome. Bilateral AOM was borderline significantly correlated with poor disease control $(P=0.05)$.

Since antibiotics prescribed in children are based on body weight, the age and weight are highly correlated (Spearman's rho $=0.88, P<0.001$ ); thus body weight was chosen instead of age in the following analyses. Using multivariate analysis, we analyzed relevant factors that influenced AOM prognosis (Table 3); it is found that, compared to patients with AOM in the spring/summer, the odds ratio (OR) of autumn/winter patients who failed control was 2.47 (95\% confidence interval (CI): 1.17-5.23, $P=0.02$ ).

4.5. Subgroup Analysis. According to the newest international growth standards for infants and children from the World Health Organization (2007) [20], with 50\% percentile estimation, children weigh about $20 \mathrm{~kg}$ at 6 years of age.
TABLE 3: Factors relevant to poor outcome of acute otitis media ( $n=$ 165 ) by multivariate analysis (binary logistic regression model).

\begin{tabular}{lcc}
\hline Variables & $P$ value & OR of failed control $(95 \% \mathrm{CI})$ \\
\hline Girls & 0.21 & $1.61(0.77-3.36)$ \\
Weight $^{*}$ & 0.48 & $0.98(0.93-1.04)$ \\
Autumn/winter & $0.02^{* *}$ & $2.47(1.17-5.23)$ \\
Bilateral AOM & 0.09 & $1.96(0.91-4.19)$ \\
Underdose & 0.58 & $1.45(0.38-5.53)$ \\
\hline
\end{tabular}

Abbreviations: AOM: acute otitis media; OR: odds ratio; CI: confidence interval. Reference groups in model: boys, illnesses in spring/summer, singlesided AOM, or high dose. * Age showed high collinearity with body weight in the model. Since antibiotics were given mainly based on body weight, weight was chosen instead of age in the regression model. ${ }^{* *} P<0.05$.

TABLE 4: Factors relevant to poor outcome of acute otitis media: subgroup analysis of children $<20 \mathrm{~kg}(n=110)$ and binary logistic regression model II.

\begin{tabular}{lcc}
\hline Variables & $P$ value & $\begin{array}{c}\text { OR of failed control } \\
(95 \% \mathrm{CI})\end{array}$ \\
\hline Girls & 0.84 & $1.10(0.44-2.74)$ \\
Weight $^{*}$ & 0.07 & $0.89(0.78-1.01)$ \\
$\begin{array}{l}\text { Autumn/winter } \\
\begin{array}{l}\text { Bilateral AOM and } \\
\text { underdose }\end{array}\end{array}$ & $0.001^{* *}$ & $4.90(1.87-12.89)$ \\
\hline
\end{tabular}

Abbreviations: AOM: acute otitis media; OR: odds ratio; CI: confidence interval. Reference groups in model: boys, illnesses in spring/summer, singlesided AOM, or high dose. * Age showed high collinearity with body weight in the model. Since antibiotics were given mainly based on body weight, weight was chosen instead of age in the regression model. ${ }^{* *} P<0.05$.

Since the daily dosage for children over $20 \mathrm{~kg}$ might surpass the equivalent common clinical dosage for adults of $1500 \mathrm{mg} /$ day, we performed subgroup analysis for 110 children who weighed less than $20 \mathrm{~kg}$ (details not shown). Among this subgroup, illness in autumn/winter was consistently a strong factor for poor prognosis (OR 4.80; 95\% CI 1.82-12.67, $P=0.002)$. In addition, the risk of failed control in bilateral AOM patients was 2.43 times more than single-sided AOM children although it was not statistically significant (95\% CI $0.90-6.53, P=0.08$ ). No obvious association was observed between under dose and AOM prognosis (OR 2.18; 95\% CI $0.38-12.48, P=0.38$ ).

When "bilateral AOM" and "under dose" were taken together, their correlation with treatment failure was evident (OR 1.63; 95\% CI 1.02-2.59, $P=0.04$ ). Again, AOM in autumn/winter was consistently associated with a poor prognosis (OR 4.90; 95\% CI 1.87-12.89, $P=0.001$ ). The results implied that the benefit of high-dose amoxicillin with clavulanate as recommended in the guidelines was more noticeable in children under $20 \mathrm{~kg}$ with bilateral AOM (Table 4).

\section{Discussion}

Our study found no significant correlation between highdose amoxicillin and better disease control when treating AOM with amoxicillin-clavulanate combination. Although 
fewer patients who were given the high dosage failed to control AOM (16.7\% for high dose versus $27.9 \%$ for under dose), the correlation was not evident. A significant association could be seen only in children below $20 \mathrm{~kg}$ with bilateral AOM when they were given insufficient dosages of amoxicillin. The OR for these children having a poor prognosis was 1.63 (95\% CI 1.02-2.59, $P=0.04$ ) in contrast to those who weighed more than $20 \mathrm{~kg}$ or with singleear disease. Illness in autumn and winter had a strongly negative impact on AOM recovery. For physicians' behavior, underdosage was extremely common (89.1\%), reflecting that the debates on dosage indeed exist even several years after the launch of the clinical practice guidelines. To a certain extent, our results were consistent with the latest guidelines revisions [21].

Many scholars questioned about guidelines recommendations being based on reports of bacterial drug resistance. Since laboratory bacteriological studies may be different from clinical trials with patients that evaluate a drug's curative effect, researchers suggest more studies evaluating clinical outcomes should be taken into consideration [1417]. There was limited evidence based solely on "dosage." This study is one of the few reports that simply investigated the relationships between amoxicillin dosage and clinical outcomes. Additional strength of our research was the precise determination of therapeutic effect. The treatment outcome (documented on medical records) regarding the eardrum status was evaluated by an otology specialist using videotelescopy coupled with a bright xenon light source which had been demonstrated to have the highest sensitivity $(97.8 \%)$ and specificity (100.0\%) [19].

Schrag's study in South America in 2001 concluded that treatment with short-course high-dosage amoxicillin (5 days, $90 \mathrm{mg} / \mathrm{kg} /$ day) reduced drug-resistant Streptococcus pneumonia (S. pneumoniae) with a probability of $8 \%$ and relative risk (RR) of $0.8(0.60-0.97)$ compared with more days with a low dosage (10 days, $40 \mathrm{mg} / \mathrm{kg} /$ day). The shortcourse high-dosage amoxicillin was especially helpful for families with 3 or more children [10]. Garrison compared the curative effect of high-dosage $(80-90 \mathrm{mg} / \mathrm{kg} /$ day $)$ and standard-dosage ( $40-45 \mathrm{mg} / \mathrm{kg} /$ day) amoxicillin, finding that the two treatments had close rates of failure ( $11 \%$ versus $12 \%$, $P=0.78$ ). In addition, the side effects of medication, number of parents' inquiry calls, parents' subjective assessment of treatment days, and number of $\mathrm{AOM}$ reoccurrences were not significantly different for the two treatments [9]. Our results were relatively close to Garrison's research. However, the children enrolled herein were older than the above-mentioned two reports and only prescriptions with amoxicillin with clavulanate were included into analysis.

The clinical practice guidelines recommendation on amoxicillin dosage is controversial. The main reasons for the higher doses $(80-90 \mathrm{mg} / \mathrm{kg} / \mathrm{day})$ in the guidelines are reports of penicillin-resistant S. pneumoniae. Before 2004, the prevalence rate of penicillin nonsusceptible $S$. pneumoniae (including intermediate and resistant strains) in the US had been increasing [22-24]. The resistance rate increased from $21 \%$ in 1995 to $25 \%$ in 1998 with a highest reported rate of $33 \%$. Strain resistant to three or more antibiotics also increased from $10 \%$ to $14 \%$. Therefore, the commonly used conventional amoxicillin dosage $(40-50 \mathrm{mg} / \mathrm{kg} /$ day $)$ was thought to be insufficient for controlling infection by drug-resistant bacteria. The committee therefore suggested that physicians should give higher doses of amoxicillin to AOM patients.

Previous studies had reported that $30 \%(15-50 \%)$ of dissociated S. pneumoniae from upper respiratory tract is not susceptible to penicillin, with a medium or high drug-resistance level. Only $S$. pneumoniae highly resistant to penicillin has no response to conventional dosages of amoxicillin $(40-50 \mathrm{mg} / \mathrm{kg} /$ day $)$. About half of nonsusceptible $S$. pneumoniae has a medium drug resistance level to penicillin (minimal inhibitory concentration (MIC): 0.1$1.0 \mu \mathrm{g} / \mathrm{mL}$ ), and the other half has a high drug-resistance level to penicillin (MIC $\geq 2.0 \mu \mathrm{g} / \mathrm{mL}$ ). Appropriately elevating dosages increase the drug concentration in middle ear fluid; theoretically, if this concentration surpasses the MIC of $S$. pneumoniae with a medium resistance level to penicillin, most cases of $S$. pneumoniae related AOM are responsive to amoxicillin treatment $[25,26]$.

As to other common pathogens in AOM, about $50 \%$ of Haemophilus influenza (H. influenza) and $100 \%$ of Moraxella catarrhalis (M. catarrhalis) are $\beta$-lactamase positive $[25$, 26]. In contrast to penicillin-resistant $S$. pneumoniae, AOM caused by $H$. influenza or $M$. catarrhalis requires a $\beta$ lactamase inhibitor such as clavulanic acid, a second- or third-generation cephalosporin, or other types of antibiotics. A sufficient dose of amoxicillin combined with clavulanic acid is effective for susceptible-to-medium-resistant $S$. pneumoniae and is also effective against $\beta$-lactamase-positive bacteria. For these reasons, the first choice of a majority of clinicians is oral amoxicillin with or without a $\beta$-lactamase inhibitor (unless patients are allergic to penicillin). In our hospital, a tertiary referral center as well as a teaching hospital, some of the specialists prescribed high-dose amoxicillin with clavulanate at the child's first visit unless patients were allergic to penicillin at the era of pneumococcal conjugate vaccination.

Another important factor that influenced prognosis was "season". Illnesses in autumn and winter were constant strongly associated with a poor prognosis in this retrospective cohort study. Both univariate and multivariate analysis showed that outcome in autumn/winter patients was poorer than spring/summer patients (OR 2.47; 95\% CI 1.17-5.23, $P=$ $0.02)$. The result was even more obvious in children $<20 \mathrm{~kg}$ (OR 4.80; 95\% CI 1.82-12.67, $P=0.002$ ). This result may be explained by a high prevalence for upper respiratory tract infections seen in autumn and winter, and people usually have several cold episodes. A child attends day care center or with siblings is likely to have multiple upper respiratory tract infections before resolution of a previous AOM, leading to more complicated disease course and poorer prognosis.

Reviewing the English literature, some reported that boys have poorer AOM prognosis than girls [27]. In our study, the ratio of boys who failed AOM primary control was lower than girls $(21.3 \%$ versus $33.8 \%)$ but was not significant $(P=0.07)$. Female gender was not an independent risk factor for poor $\mathrm{AOM}$ control either in univariate or multivariate analysis (OR 1.61; $95 \%$ CI $0.77-3.36, P=0.21$ ). It had been reported that 
bilateral infection gave rise to poor prognosis [27]. Our study concluded that patients who had single-ear AOM initially had $19.8 \%$ failed control, as opposed to $33.3 \%$ for bilateral infection patients, although it was borderline significant $(P=$ $0.05)$. This may be explained by insufficient samples $(n=165)$ in this research. Other parameters related to illness severity such as body temperature and earache, or infant crying, could not be comprehensively collected during the process of chart review and were thus not incorporated into outcome analysis.

Patients in medical centers might have different or complicated pathogens causing AOM more than patients from community groups, and the possibility of drug-resistant bacteria could be higher. Children enrolled in this study were from the ENT Department in our hospital, a medical center. Besides, the average age in our cohort was 4.88 years old, which is somewhat older than the commonly seen age for AOM. Older children should have a lower chance of AOM [28] because of relative maturity of the Eustachian tubemiddle ear ventilation system; but on the contrary, patients who are over the common age and had middle ear infections might have more complex illnesses. These two perspectives could have led to the result of a comparable curative effect of regular-dose and high-dose amoxicillin with clavulanate.

In real-world settings, physicians prescribe antibiotics based on their understanding of the pathogenesis, course of disease, severity of the illness, pathogens causing the illness, and pharmacological knowledge. Other factors including physician's personal preference, bacterial drug-resistance reports from different areas or medical associations, caregivers' attitude, and insurance benefits may also play a role.

Our study has limitations. This is a retrospective cohort study; some of the possible risk factors such as past AOM history, previous visit to primary physicians, medication that had been used, day care attendance, siblings number, smoking in the household, and breast milk feeding could not be fully collected while doing chart review. The compliance for antibiotics was not known clearly either.

\section{Conclusion}

Our study demonstrated that high-dose amoxicillin with clavulanate as recommended in AOM clinical practice guidelines provided significant benefits only in children under $20 \mathrm{~kg}$ with bilateral illnesses. The question of how amoxicillin (amoxicillin-potassium clavulanate combination) dosage affects the prognosis of AOM needs more prospective controlled studies that comprehensively collect common prognostic factors with a large number of samples and laboratory bacteriological analyses.

\section{Abbreviations}

$\begin{array}{ll}\text { AOM: } & \text { Acute otitis media } \\ \text { CI: } & \text { Confidence interval } \\ \text { H. influenza: } & \text { Haemophilus influenza } \\ \text { M. catarrhali: } & \text { Moraxella catarrhalis } \\ \text { MIC: } & \text { Minimal inhibitory concentration } \\ \text { OME: } & \text { Otitis media with effusion }\end{array}$

OR: $\quad$ Odds ratio

S. pneumonia: Streptococcus pneumonia.

\section{Conflict of Interests}

All authors have no financial relationships relevant to this paper to disclose. All authors report no conflict of interests.

\section{Acknowledgment}

The study project was approved by Institutional Review Board of Taipei Veterans General Hospital (VGHIRB no. 2012-09027B).

\section{References}

[1] V. M. Freid, D. M. Makuc, and R. N. Rooks, "Ambulatory health care visits by children: principal diagnosis and place of visit," Vital and Health Statistics, no. 137, pp. 1-23, 1998.

[2] M. M. Rovers, A. G. M. Schilder, G. A. Zielhuis, and R. M. Rosenfeld, "Otitis media," The Lancet, vol. 363, no. 9407, pp. 465-473, 2004.

[3] P. S. Mattila, "Antibiotics in childhood acute otitis media," The Lancet, vol. 368, no. 9545, pp. 1397-1398, 2006.

[4] F. Zhou, A. Shefer, Y. Kong, and J. P. Nuorti, "Trends in acute otitis media-related health care utilization by privately insured young children in the United States, 1997-2004," Pediatrics, vol. 121, no. 2, pp. 253-260, 2008.

[5] D. K. Cherry and D. A. Woodwell, "National ambulatory medical care survey: 2000 summary," Advance Data, no. 328, pp. 1-32, 2002.

[6] J. H. Wolleswinkel-van den Bosch, E. A. Stolk, M. Francois, R. Gasparini, and M. Brosa, "The health care burden and societal impact of acute otitis media in seven European countries: results of an Internet survey," Vaccine, vol. 28, no. 6, pp. G39G52, 2010.

[7] R. Alsarraf, C. J. Jung, J. Perkins, C. Crowley, N. W. Alsarraf, and G. A. Gates, "Measuring the indirect and direct costs of acute otitis media," Archives of Otolaryngology, vol. 125, no. 1, pp. 12$18,1999$.

[8] "Diagnosis and management of acute otitis media," Pediatrics, vol. 113, no. 5, pp. 1451-1465, 2004.

[9] G. D. Garrison, P. C. Sorum, W. Hioe, and M. M. Miller, "Highdose versus standard-dose Amoxicillin for acute otitis media," Annals of Pharmacotherapy, vol. 38, no. 1, pp. 15-19, 2004.

[10] S. J. Schrag, C. Peña, J. Fernández et al., "Effect of short-course, high-dose amoxicillin therapy on resistant pneumococcal carriage: a randomized trial," Journal of the American Medical Association, vol. 286, no. 1, pp. 49-56, 2001.

[11] I. Brook and A. E. Gober, "The effects of treatment of acute otitis media with a low dose vs a high dose of amoxicillin on the nasopharyngeal flora," Archives of Otolaryngology, vol. 135, no. 5, pp. 458-461, 2009.

[12] J. R. Casey, S. L. Block, J. Hedrick, A. Almudevar, and M. E. Pichichero, "Comparison of amoxicillin/clavulanic acid high dose with cefdinir in the treatment of acute otitis media," Drugs, vol. 72, no. 15, pp. 1991-1997, 2012.

[13] A. Arguedas, C. Soley, B. J. Kamicker, and D. M. Jorgensen, "Single-dose extended-release azithromycin versus a 10-day regimen of amoxicillin/clavulanate for the treatment of children 
with acute otitis media," International Journal of Infectious Diseases, vol. 15, no. 4, pp. e240-e248, 2011.

[14] E. R. Wald, "Acute otitis media: more trouble with the evidence," Pediatric Infectious Disease Journal, vol. 22, no. 2, pp. 103-104, 2003.

[15] S. L. Woolley and D. R. K. Smith, "Acute otitis media in children-there are guidelines but are they followed?" Journal of Laryngology and Otology, vol. 119, no. 7, pp. 524-528, 2005.

[16] E. K. Vouloumanou, D. E. Karageorgopoulos, M. S. Kazantzi, A. M. Kapaskelis, and M. E. Falagas, "Antibiotics versus placebo or watchful waiting for acute otitis media: a meta-analysis of randomized controlled trials," Journal of Antimicrobial Chemotherapy, vol. 64, no. 1, pp. 16-24, 2009.

[17] L. Vernacchio, R. M. Vezina, and A. A. Mitchell, "Knowledge and practices relating to the 2004 acute otitis media clinical practice guideline: a survey of practicing physicians," Pediatric Infectious Disease Journal, vol. 25, no. 5, pp. 385-389, 2006.

[18] S. Thanaviratananich, M. Laopaiboon, and P. Vatanasapt, "Once or twice daily versus three times daily amoxicillin with or without clavulanate for the treatment of acute otitis media," Cochrane Database of Systematic Reviews, no. 4, p. CD004975, 2008.

[19] A.-S. Shiao and Y.-C. Guo, "A comparison assessment of videotelescopy for diagnosis of pediatric otitis media with effusion," International Journal of Pediatric Otorhinolaryngology, vol. 69, no. 11, pp. 1497-1502, 2005.

[20] Organization WH, "The WHO child growth standards," 2007, http://www.who.int/growthref/who2007_weight_for_age/en /index.html.

[21] A. S. Lieberthal, A. E. Carroll, T. Chonmaitree et al., "The diagnosis and management of acute otitis media," Pediatrics, vol. 131, no. 3, pp. e964-e999, 2013.

[22] S. F. Dowell, J. C. Butler, and G. S. Giebink, "Acute otitis media: management and surveillance in an era of pneumococcal resistance. Drug-Resistant Streptococcus pneumoniae Therapeutic Working Group," The Pediatric Infectious Disease Journal, vol. 18, no. 1, pp. 1-9, 1999.

[23] C. G. Whitney, M. M. Farley, J. Hadler et al., "Increasing prevalence of multidrug-resistant Streptococcus pneumoniae in the United States," The New England Journal of Medicine, vol. 343, no. 26, pp. 1917-1924, 2000.

[24] J. R. Casey and M. E. Pichichero, "Changes in frequency and pathogens causing acute otitis media in 1995-2003," Pediatric Infectious Disease Journal, vol. 23, no. 9, pp. 824-828, 2004.

[25] M. R. Jacobs, S. Bajaksouzian, A. Zilles, G. Lin, G. A. Pankuch, and P. C. Appelbaum, "Susceptibilities of Streptococcus pneumoniae and Haemophilus influenzae to 10 oral antimicrobial agents based on pharmacodynamic parameters: 1997 U.S. surveillance study," Antimicrobial Agents and Chemotherapy, vol. 43, no. 8, pp. 1901-1908, 1999.

[26] M. R. Jacobs, D. Felmingham, P. C. Appelbaum et al., "The Alexander project 1998-2000: susceptibility of pathogens isolated from community-acquired respiratory tract infection to commonly used antimicrobial agents," Journal of Antimicrobial Chemotherapy, vol. 52, no. 2, pp. 229-246, 2003.

[27] M. E. Pichichero and J. R. Casey, "Acute otitis media: making sense of recent guidelines on antimicrobial treatment," Journal of Family Practice, vol. 54, no. 4, pp. 313-322, 2005.

[28] K. Revai, L. A. Dobbs, S. Nair, J. A. Patel, J. J. Grady, and T. Chonmaitree, "Incidence of acute otitis media and sinusitis complicating upper respiratory tract infection: the effect of age," Pediatrics, vol. 119, no. 6, pp. e1408-e1412, 2007. 


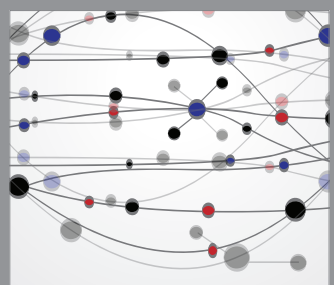

The Scientific World Journal
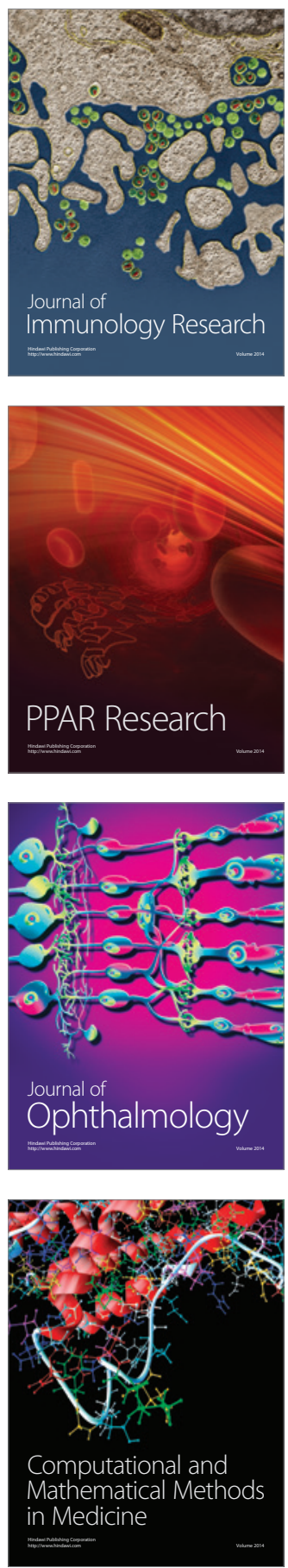

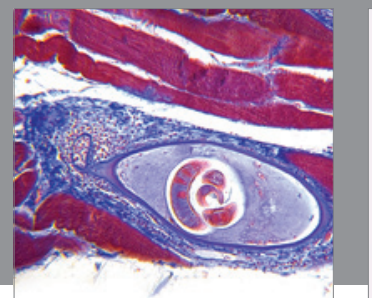

Gastroenterology

Research and Practice
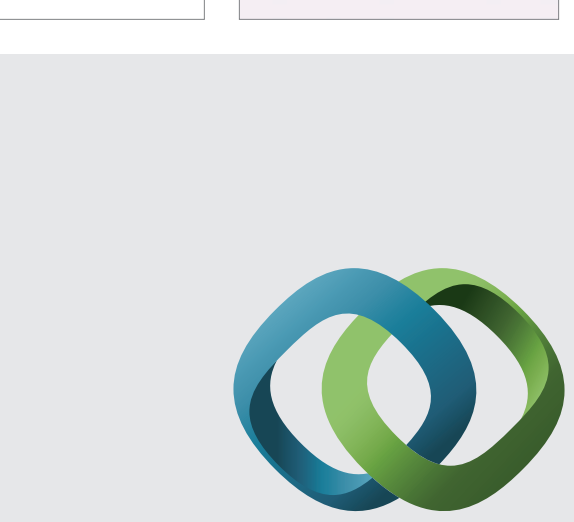

\section{Hindawi}

Submit your manuscripts at

http://www.hindawi.com
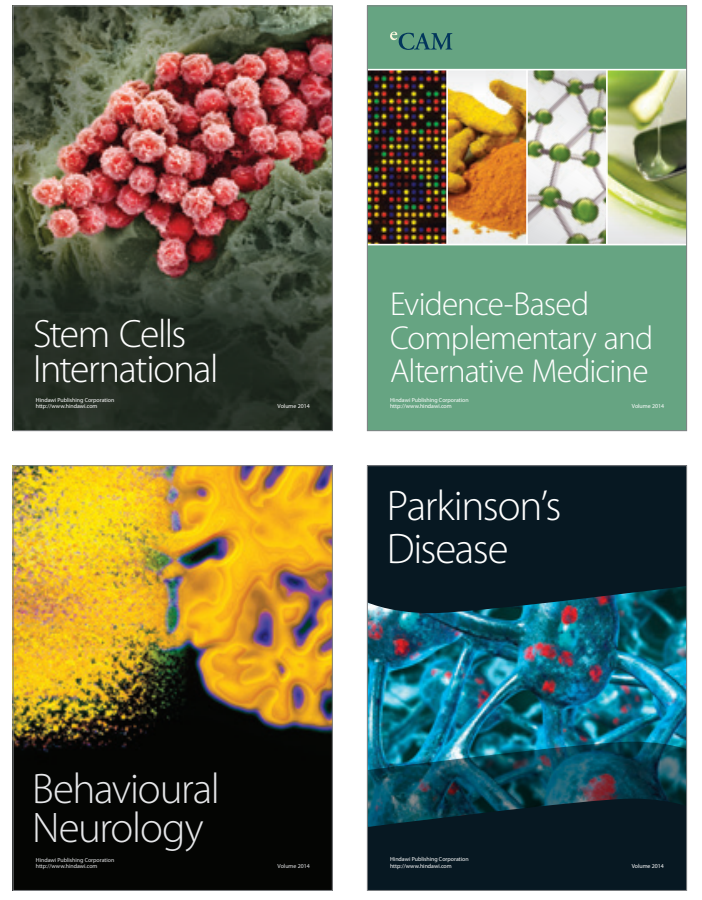
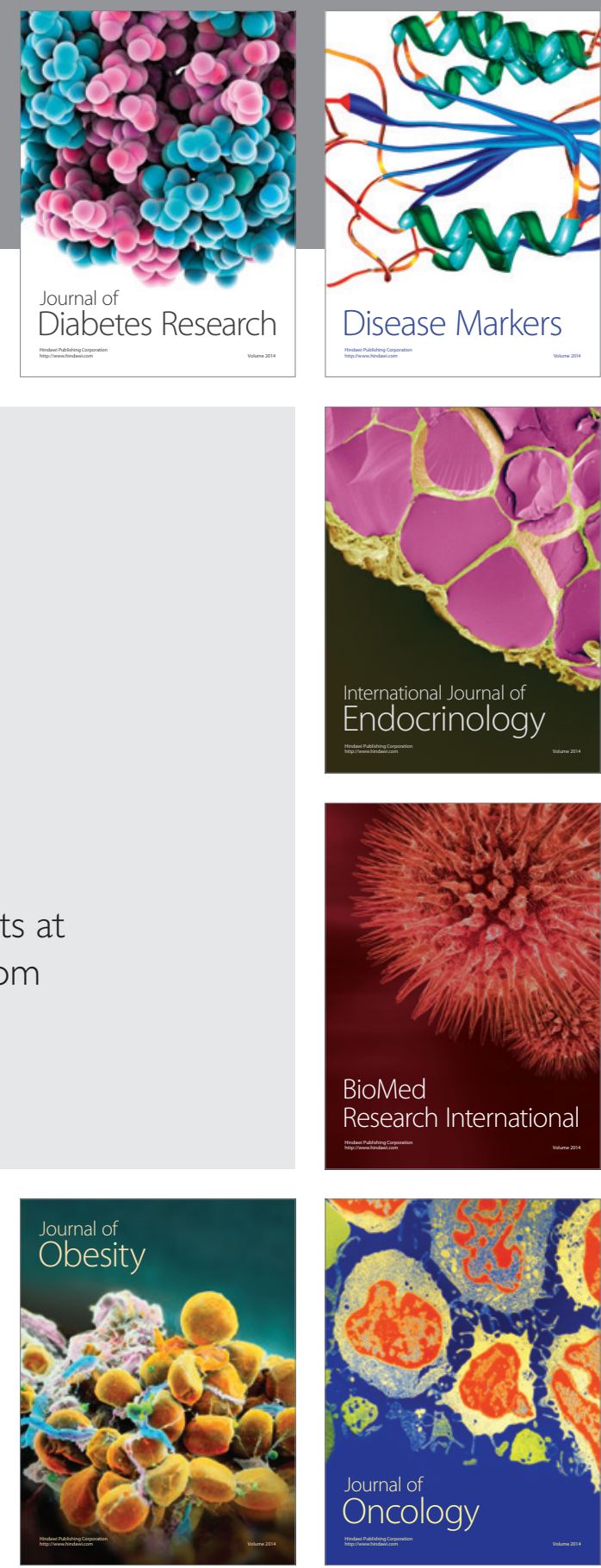

Disease Markers
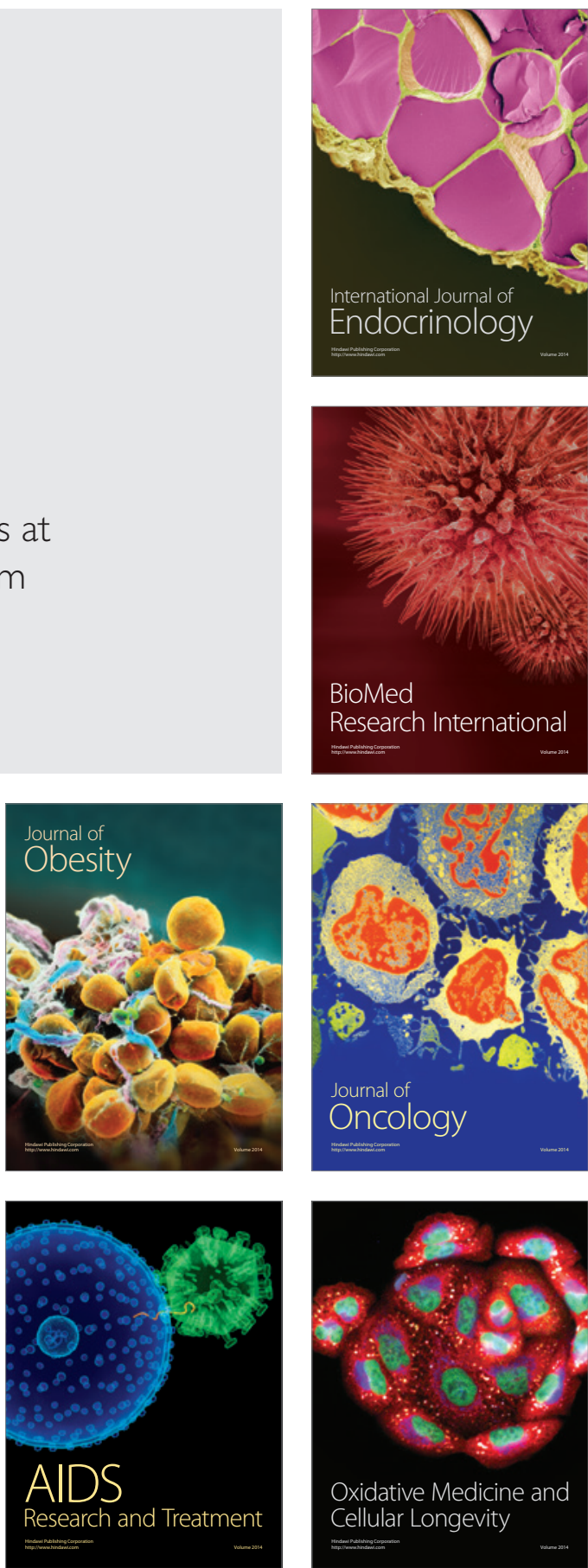